\title{
TWO-GENERATOR GROUPS II
}

\section{J.L. BRenner and James Wiegold}

\begin{abstract}
Let $n$ be an odd integer greater than 9 . It is proved that the alternating group $A_{n}$ has spread 3 in the sense that for any non-trivial elements $x_{1}, x_{2}, x_{3}$ of $A_{n}$, there is an element $y$ in $A_{n}$ such that $\left\langle x_{i}, y\right\rangle=A_{n}$ for $i=1,2,3$.
\end{abstract}

\section{Introduction}

This article is a continuation of [2], to which the reader is referred for motivation and background material. We recall the principal definition.

DEFINITION 1.1. A finite group $G$ is said to have spread $r$ if for every set $\left\{x_{1}, \ldots, x_{p}\right\}$ of non-trivial elements of $G$ there is an element $y$ such that $\left\langle x_{i}, y\right\rangle=G$ for $i=1,2, \ldots, r$. The set of all finite groups having spread $r$ is denoted by $\Gamma_{r}$.

Thus each $\Gamma_{r}$ is a set of two-generator groups, and $\Gamma_{r} \supseteq \Gamma_{p+l}$ for all $r \geq 1$. In [2] (see also Binder [1] for an independent proof), we showed that $A_{2 n} \in \Gamma_{4} \backslash \Gamma_{5}$ for $n \geq 4$, and various analogous results for the groups $\operatorname{PSL}(2, q)$. On the other hand, it turned out that $A_{19}$ has enormous spread, more than $6,000,000,000$ or so. This is due to the scarcity of transitive subgroups of $A_{19}$, and we indicate here (in \$4) a similar result for alternating groups $A_{p}$, whenever $p$ is a prime for

Received 19 December 1979. 
which $A_{p}$ displays the same scarcity of transitive subgroups. This result, and general considerations, lead us to believe that $A_{n}$ has spread that tends to infinity with $n$, for odd $n$. We are unable to prove anything like this. Combinatorial methods like those in [2] are used to prove (in \$2) that $A_{n} \in \Gamma_{3}$ if $n$ is odd and at least 11 . There is no doubt that similar arguments would prove that $A_{n} \in \Gamma_{4}$ for sufficiently large $n$, or even $A_{n} \in \Gamma_{5}$ and so on, but the extra combinatorial complication is hardly worth indulging in, given our belief that the spread of $A_{n}$ is unbounded for large odd $n$.

For odd composite $n$, we give a very simple proof in $\$ 3$ that $A_{n}$ does not have spread $C_{d-1}^{n-1}$, where $d$ is the smallest prime divisor of $n$. This is very easy. Quite possibly the following problem will have an affirmative answer, but it will be exceedingly difficult to prove.

PROBLEM 1.2. For $n$ odd and composite, with $d$ the smallest prime factor, does $A_{n}$ have spread close to $c_{d-1}^{n-1}-1$ ?

We have been unable to resolve this even in the simplest case, namely for $A_{9}$. Finally, in $\S 3$, we sketch a proof of the (special but new) result that $\operatorname{PSL}(3,4)$ cannot be generated by an element of order 2 and one of order 3 . The context is the following. In [2] we defined $\Gamma_{1}^{(k)}$ to consist of those groups $G$ in $\Gamma_{1}$ such that every non-trivial element $a$ belongs to a generating pair $\{a, b\}$ such that one of $a, b$ has order $k$. Thus $\operatorname{PSL}(3,4) \notin \Gamma_{1}^{(2)}$. In an interesting doctoral dissertation [4], Langer filled more of the gaps in [2] by establishing that, for $q \neq 2,9$, the group $\operatorname{PSL}(2, q)$ belongs to $\Gamma_{l}^{(k)}$ whenever it has an element of order $k$.

\section{Alternating groups of odd degree}

We mentioned in [2] that $A_{5} \in \Gamma_{2} \backslash \Gamma_{3}$. It is easy to prove that $A_{7}$ 
and $A_{9}$ lie in $\Gamma_{2}$, and we shall omit the proof. The reader will see how to do it by examining the methods employed in this section.

Wherever possible we shall use "standard elements" as supplementary generators.

DEFINITION 2.1. Let $n=2 k+1, k>2$. A standard type is the type $k^{1}\left(\frac{1}{2}(k+1)\right)^{2}$ if $k$ is odd; $(k+1)^{1}\left(\frac{1}{2} k\right)^{2}$ if $k$ is even. The elements in $A_{n}$ of standard type are called standard elements.

Thus for odd $k$, a standard element is a product of a $k$-cycle and two $\frac{1}{2}(k+1)$-cycles, and similarly for even $k$. This is the vital property of standard elements:

THEOREM 2.2. For odd $n>5$, the only transitive subgroup of $A_{n}$ containing a standard element is $A_{n}$ itself.

Proof. Any subgroup $H$ containing a standard element contains a $k$-cycle if $k$ is odd and a $(k+1)$-cycle if $k$ is even. Since $k$ and $k+1$ are prime to $2 k+1$, this means that $H$ is primitive if it is transitive. Now use a theorem of Williamson ([7]; see also [2]): if a primitive group $G$ of degree $n$ contains a $t$-cycle, $1<t<n$, then $G \supseteq A_{n}$ unless $t>(n-t) !$.

To prove that every three elements of $A_{n}$ have a common "mate", we may assume at the outset that they are of prime order. In the proof of the theorem that follows, we do not go into every detail, to save space and to spare the reader. In all cases where this is possible, we use a standard element as common mate: when it is not possible, an n-cycle always works. Indeed, one of the things that makes the odd-degree case so difficult to contend with is that sometimes we are forced to use an $n$-cycle as common mate; for example, only an $n$-cycle will mate each of $(1,2)(3,4)$, $(1,3)(2,4),(1,4)(2,3)$ simultaneously.

THEOREM 2.3. Let $x_{1}, x_{2}, x_{3}$ be elements of prime order in $A_{n}, n$ odd and greater than 9 , with the order of $x_{i}$ greater than or equal to the order of $x_{j}$ if $i \leq j$. Then there is a standard element $y$ such that $\left\langle x_{i}, y\right\rangle=A_{n}$ for $i=1,2,3$, except in the following cases: 
(1) $x_{1}, x_{2}$ are 3-cycles moving exactly 2 symbols in common, say $x_{1}=(1,2,3), x_{2}=(1,2,4)$, and $x_{3}$ is of the form $(3,4, \gamma)$ or $(3,4)(\alpha, \beta)$;

(2) $x_{1}, x_{2}$ are 3 -cycles moving exactly one symbol in common, say $x_{1}=(1,2,3), x_{2}=(1,4,5)$, and $x_{3}$ is of the form $(2,3)(4,5),(2,4)(3,5)$ or $(2,5)(3,4)$;

(3) $x_{1}, x_{2}, x_{3}$ are all of order 2 , and move exactly the same four symbols, say $x_{1}=(1,2)(3,4), x_{2}=(1,3)(2,4)$, $x_{3}=(1,4)(2,3)$.

In each of the exceptional cases, there is an n-cycle that is a common mate for $x_{1}, x_{2}, x_{3}$.

Proof. We split the proof up into several cases. Recall that each cycle in every standard element moves at least 3 symbols, since $n \geq 11$.

CASE 1. $x_{1}, x_{2}$ have odd order.

Case 1A. Suppose that there is an orbit $\Omega_{1}$ of $\left\langle x_{1}\right\rangle$ and an orbit $\Omega_{2}$ of $\left\langle x_{2}\right\rangle$ with subsets $T_{1} \subseteq \Omega_{1}, T_{2} \subseteq \Omega_{2}$ such that $T_{1} \cap T_{2}=\emptyset$ and $\left|T_{1}\right|=\left|T_{2}\right|=3$. For simplicity write $T_{1}=\{1,2,3\}, T_{2}=\{4,5,6\}$. Thus $1,2,3$ occur in the same cycle of $x_{1}$, and $4,5,6$ in the same cycle of $x_{2}$, and by Theorem 2.2 any standard element

$$
y=\left(1, \alpha_{1}, \ldots\right)\left(2, \alpha_{2}, \ldots\right)\left(3, \alpha_{3}, \ldots\right) \text {, }
$$

where $\alpha_{1}, \alpha_{2}, \alpha_{3}$ are $4,5,6$ in some order, will be a common mate for $x_{1}$ and $x_{2}$, since $\left\langle x_{1}, y\right\rangle$ and $\left\langle x_{2}, y\right\rangle$ are transitive. Clearly, if $x_{3}$ has odd order, then three symbols from the same cycle of $x_{3}$ can be disposed, one in each of the three cycles in one of the six choices of $y$; and this choice will be a common mate for all three elements however the remaining symbols are distributed. The same sort of reasoning applies if $x_{3}$ is of order 2 . 
Case 1B. If the condition imposed on the orbits in Case 1A does not hold, then for every orbit $\Omega_{1}$ of $\left(x_{1}\right\rangle$ and every orbit $\Omega_{2}$ of $\left(x_{2}\right)$, every three-element subset of $\Omega_{1}$ intersects every three-element subset of $\Omega_{2}$. This imposes severe restrictions on $\Omega_{1}, \Omega_{2}$. A moment's thought shows that one of three things must happen:

(i) $\left|\Omega_{1}\right|=\left|\Omega_{2}\right|=3$ and $\Omega_{1} \cap \Omega_{2} \neq \emptyset$;

(ii) $\left|\Omega_{1}\right|=5,\left|\Omega_{2}\right|=3$ and $\Omega_{2} \subseteq \Omega_{1}$, or symetrically

$$
\left|\Omega_{1}\right|=3,\left|\Omega_{2}\right|=5, \Omega_{1} \subseteq \Omega_{2} \text {; }
$$

(iii) $\Omega_{1}=\Omega_{2}$ and $\left|\Omega_{1}\right|=5$.

Since these conditions hold for every pair of orbits, $x_{1}$ and $x_{2}$ must have very restricted forms. If $x_{1}$ has order more than 3 , then it follows that $x_{1}$ is a 5-cycle and $x_{2}$ is a 5-eycle or a 3-cycle with support contained in that of $x_{1}$. In other words, $x_{1}$ is of the form $(1,2,3,4,5)$ say, and $x_{2}$ is either a 5-cycle on the same letters or a 3-cycle on three of them, say on 1, 2, 3 with no loss of generality. In this case a standard element

$$
y=(1, \ldots)(2, \ldots)(3, \ldots)
$$

will be a mate for $x_{1}$ and $x_{2}$. Clearly, whatever $x_{3}$ is, $y$ can be fleshed out to be a mate for $x_{3}$ too.

Thus we may now suppose that $x_{1}$ and $x_{2}$ have order 3 , and that every cycle in $x_{1}$ intersects every cycle in $x_{2}$. This can happen in very few ways. Neither element can move more than 9 symbols, and we may assume that $9 \geq\left|\operatorname{supp}\left(x_{1}\right)\right| \geq\left|\operatorname{supp}\left(x_{2}\right)\right|$.

If $x_{1}$ moves 9 symbols, the situation is typified by

$$
\begin{aligned}
& x_{1}=(1,2,3)(4,5,6)(7,8,9), \\
& x_{2}=(1,4,7) \ldots,
\end{aligned}
$$


in which case any standard element

$$
y=(1, \ldots)(4, \ldots)(7, \ldots)
$$

is a common mate for $x_{1}$ and $x_{2}$, and it can be fleshed out to be a mate for $x_{3}$, whatever $x_{3}$ may be other than $(1,4,7)^{ \pm 1}$. If $x_{3}$ is $(1,4,7)^{ \pm 1}$, we can assume that $x_{2}$ has a further 3-cycle in its decomposition, say $(2,5,8)$ or its like, and argue from there.

If $x_{1}$ moves 6 symbols, one has cases like

$$
\begin{aligned}
& x_{1}=(1,2,3)(4,5,6), x_{2}=(1,4, \alpha)(2,5, \beta), \\
& x_{1}=(1,2,3)(4,5,6), x_{2}=(1,4, \alpha) .
\end{aligned}
$$

In these cases a standard element

$$
\begin{aligned}
& (1, \ldots)(4, \ldots)(\alpha, \ldots) \quad \text { if } \alpha \in\{2,3,5,6\}, \\
& (1, \ldots)(4, \ldots)(\alpha, \gamma, \ldots) \text { if } \alpha \notin\{2,3,5,6\},
\end{aligned}
$$

for any $\gamma \in\{2,3,5,6\}$, is a common mate for $x_{1}, x_{2}$. Clearly, whatever $x_{3}$ may be, there is room to accommodate $x_{3}$ as well.

$$
\text { Lastly, if }\left|\operatorname{supp}\left(x_{1}\right)\right|=3 \text {, then both } x_{1} \text { and } x_{2} \text { are 3-cycles, }
$$
and problematical cases are typified by

$$
\begin{aligned}
& x_{1}=(1,2,3), x_{2}=(1,2,4), \\
& x_{1}=(1,2,3), x_{2}=(1,4,5),
\end{aligned}
$$

the case $x_{1}=x_{2}^{ \pm 1}$ being trivial.

In the first case, a standard common mate for $x_{1}, x_{2}$ has to be of a form like

$$
(1, \ldots)(2, \ldots)(3, \ldots, 4, \ldots)
$$

This will not mate any elements $(\alpha, \beta)(3,4)$ nor $(3,4, \gamma)^{ \pm 1}$, but it can be fleshed-out to mate anything else. We have thus come to our first exceptions, and we must provide our $n$-cycle common mate. Evidently, the 
essentially different possibilities for $x_{3}=(\alpha, \beta)(3,4)$ are $(1,2)(3,4),(1,5)(3,4),(5,6)(3,4)$; in all cases $(1,2, \ldots, n)$ is a common mate for $(1,2,3),(1,2,4),(\alpha, \beta)(3,4)$. Similarly, the essentially different cases for $x_{3}=(3,4, \gamma)^{ \pm 1}$ are $(2,3,4)^{ \pm 1}$ and $(3,4,5)^{ \pm 1}$; and again $(1,2, \ldots, n)$ is a common mate. The necessary calculations are routine, and we omit them.

In the second case, a standard common mate for $x_{1}, x_{2}$ must take one of these forms

$$
\begin{aligned}
& g=(1, \ldots)(2, \ldots, 4, \ldots)(3, \ldots, 5, \ldots), \\
& h=(1, \ldots)(2, \ldots, 5, \ldots)(3, \ldots, 4, \ldots),
\end{aligned}
$$

and only $(2,3)(4,5),(2,5)(3,4),(2,4)(3,5)$ cannot be mated by one of $g, h$. But yet again, $(1,2, \ldots, n)$ is a common mate in all problematical cases.

We have now finished the case in which $x_{1}, x_{2}$ have odd order.

CASE 2. $x_{1}$ has odd order, $x_{2}, x_{3}$ have order 2 .

Case 2A. Suppose that there are 3 entries in a cycle of $x_{1}$, say $1,2,3$, different from the entries in two cycles of $x_{2}$, say

$4,5,6,7$; so

$$
\begin{aligned}
& x_{1}=(1, \ldots, 2, \ldots, 3, \ldots) \ldots, \\
& x_{2}=(45)(67) \ldots .
\end{aligned}
$$

There are essentially four distributions of $1,2,3,4,5,6,7$ among the three cycles of a standard element $y$ that ensures that it mates $x_{1}$ and $x_{2}$. Here are the four with $1,2,3$ in a given order:

$$
\begin{aligned}
& (1,4,6, \ldots)(2,5, \ldots)(3,7, \ldots), \\
& (1,4,7, \ldots)(2,5, \ldots)(3,6, \ldots), \\
& (1,4, \ldots)(2,5,6, \ldots)(3,7, \ldots), \\
& (1,4, \ldots)(2,5,7, \ldots)(3,6, \ldots) .
\end{aligned}
$$


Clearly, whatever element of order two $x_{3}$ might be, one of the 24 possibilities for $y$ can be fleshed out so as to mate it.

Case 2B. Suppose that the condition assumed in Case $2 \mathrm{~A}$ is false. As in Case $1 B$, we get strong restrictions on the possibilities for $x_{1}, x_{2}$. For a start, $x_{1}$ has order less than or equal to 5 ; if it has order 5 it is a 5-cycle and both $x_{2}, x_{3}$ have support of order 4 intersecting every three-element subset of $\operatorname{supp}\left(x_{1}\right)$. In other words, $x_{1}$ is something like $(1,2,3,4,5)$, and each of $x_{2}, x_{3}$ something like $(1,2)(3,4)$ or $(1,2)(3,6)$. This case is easy; just start with a tentative common mate for $x_{2}, x_{3}$ and flesh it out to accommodate $x_{1}$.

Suppose finally that $x_{1}$ has order 3 , say

$$
x_{1}=(1,2,3) \ldots .
$$

Obviously, $x_{2}\left[x_{3}\right]$ (being even) cannot move more than 8 symbols; for if it did, it would have $\geq 6$ transpositions, and some pair of them would fail to involve any of $1,2,3$. It is possible for $x_{2}\left[x_{3}\right]$ to have support of cardinal 8 , but then $x_{1}$ has support of cardinal less than or equal to 6 ; and the usual sort of argument can be pushed through to find a standard common mate.

CASE $3 . x_{1}, x_{2}, x_{3}$ are all of order 2.

By now the plot should be clear. One first considers the case where $x_{1}, x_{2}$ have forms like

$$
\begin{aligned}
& x_{1}=(1,2)(3,4) \ldots, \\
& x_{2}=(5,6)(7,8) \ldots .
\end{aligned}
$$

The symbols $1,2,3,4,5,6,7,8$ can then be disposed in 32 essentially different ways in a "standard shape", so that any fleshing-out is a common mate for $x_{1}, x_{2}$. In all cases there is enough flexibility to accommodate $x_{3}$ as well. 
If $x_{1}, x_{2}$ do not have shapes as indicated, there are clearly strong restrictions, and the whole argument goes through to provide a standard common mate except when $x_{1}, x_{2}, x_{3}$ have forms like $(1,2)(3,4)$, $(1,3)(2,4),(1,4)(2,3)$ respectively. In that case $(1,2, \ldots, n)$ is a common mate, as is easy to check.

We can thus state:

COROLLARY 2.4. $A_{n}$ has spread 3 for $n \geq 11$.

\section{The case of odd composite $n$}

Here is the simple result mentioned in the introduction.

THEOREM 3.1. Let $n$ be an odd composite integer, and $d$ its smallest proper divisor. Then $A_{n}$ does not have spread $z=C_{d-1}^{n-1}$.

For each choice of $d-1$ symbols out of the $n-1$ symbols $2,3, \ldots, n$, we construct a $d$-cycle moving 1 and these $d-1$ chosen symbols. There are $z$ such $d$-cycles, and we claim that no common mate exists. Firstly, a common mate must be an $n$-cycle, since any element with more than one cycle in its canonical decomposition generates an intransitive subgroup with one of our $d$-cycles. However, every $n$-cycle generates an imprimitive group with one of them.

This is an inadequate sort of result, but it is the best we can do. For the remainder of the section we sketch a proof that $\operatorname{PSL}(3,4)$ cannot be generated by an element of order 2 and one of order 3 .

The following argument has been kindly supplied by Professor A. Sinkov; it replaces our original argument, which was longer. Since $\operatorname{PSL}(3,4)$ has no element of order greater than 7 , two elements of orders 2,3 in $\operatorname{PSL}(3,4)$ must generate $(2,3, n ; p)$ with $n \leq 7$ and $p \leq 7$ [7]. All these groups are known. In fact $(2,3,7 ; 7)$ has order 1092 ; see [3]. This shows that $\operatorname{PSL}(3,4)$ cannot in fact be generated by elements of orders 2,3 .

\section{The spread of $A_{p}, p$ prime}

In this section we show that for certain primes $p$, the spread of $A_{n}$ 
is huge. We call $p$ a good prime if the only insoluble transitive groups of degree $p$ are $A_{p}$ and $S_{p}$. Neumann and Ligler [5] listed 19 such primes, and reported the existence of many others. (19 is one of the good primes.) Undoubtedly the consensus now is that the good primes are in the majority.

Let $p$ be a good prime, and $q$ a prime dividing $\frac{z}{2}(p-1)$. The group $A_{p}$ contains $(p-1) ! p$-cycles that lie in $(p-2) !$ cyclic groups $\left\langle v_{\nu}\right\rangle=T_{\nu}$ of order $p$, the sylow $p$-subgroups of $A_{p}$. In the symmetric group $S_{p}$, there is a $(p-l)$-cycle $w_{v}$ that transforms $v_{v}$ into its $g$ th power, where $g$ is a primitive root $\bmod p$. Thus the normalizer $N_{\nu}$ of $T_{v}$ in $A_{p}$ is an extension of $T_{v}$ by a cyclic group $\left\langle u_{v}\right\rangle$ of order $\frac{1}{2}(p-1)$. This normalizer contains elements of order $q$. Each such element $x$ is the product of $(p-1) / q q$-cycles, and we need to know the number $r=r(p, q)$ of sylow $p$-normalizers $N_{\nu}$ that contains a particular element $x$ of this shape $q^{(p-1) / q}$.

The total number of elements in $A_{p}$ that have the same cyclestructure as $x$ is $p ! / s(p, q)$, where $s(p, q)=q^{(p-1) / q}((p-1) / q) !$. of these, $p(q-1)$ lie in each of the $(p-2)$ ! Sylow $p$-normalizers $N_{v}$. This establishes the relation

$$
p(q-1)(p-2) !=r(p, q) \cdot p ! / s(p, q),
$$

from which the value of $r(p, q)$ is determined. It is easily checked that for fixed $p, r(p, q)$ is maximal when $q$ is the smallest prime divisor of $\frac{1}{2}(p-1)$.

THEOREM 4.2. If $p$ is a good prime, then $A_{p}$ has spread $t_{p}-1$, where

$$
t_{p}=(p-1) ! /(s(p, q)(q-1)),
$$

and $q$ is the smallest prime divisor of $\frac{2}{2}(q-1)$.

Proof. We use the same sort of argument as was used in [2] for the case $p=19$. Let $t_{p}-1$ non-trivial elements $x_{i}$ be given. We shall 
find a common mate $y$ for them, and indeed $y$ will be a p-cycle. For any $x_{i}$ that lies in no normalizer $N_{v}$, there is considerable freedom. In fact $\left\langle x_{i}, y\right\rangle$ is insoluble and transitive in such a case, whatever $p$-cycle $y$ is chosen to be; and thus $\left\langle x_{i}, y\right\rangle=A_{p}$ since $p$ is a good prime. For the other elements $x_{i}$ the reasoning is more delicate. If $x_{i}$ is a p-cycle it lies in just one p-normalizer. If $x_{i}$ has type $l \times \ldots \times l$, where $l$ is a prime divisor of $\frac{l}{2}(p-l)$, then $x_{i}$ lies in $r(p, \tau)$ normalizers $N_{\nu}$. For fixed $p$, the largest value of $r(p, \tau)$ is taken when $Z=q$, the smallest prime divisor of $\frac{1}{2}(p-1)$. By (4.1), the number of $N_{v}$ involved does not exceed

$$
\begin{aligned}
r(p, q)\left(t_{p}-1\right)<r(p, q) t_{p} & =\frac{p(q-1)(p-2) ! s(p, q)}{p !} \frac{(p-1) !}{s(p, q)(q-1)} \\
& =(p-2) !
\end{aligned}
$$

Hence there is a p-cycle left over to play the role of $y$.

COROLLARY 4.3. If there are infinitely many good primes, then the alternating groups of prime degree have unbounded spread.

In the opposite direction, we have the following result:

THEOREM 4.4. If $p>5$, then $A_{p}$ does not have spread $t_{p}+3$.

Proof. As above, let $q$ be the smallest prime factor of $\frac{1}{2}(p-1)$; let $z_{i}$ be a collection of $t_{p}$ elements of type $q \times q \times \ldots \times q$ (that is, $(p-1) / q$ q-cycles $)$, chosen so that each one lies in exactly $r(p, q)$ normalizers $N_{v}$, and so every normalizer $N_{v}$ contains one of the $z_{i}$.

Further, set

$$
z_{t_{p}+1}=(1,2)(3,4), z_{t_{p}+2}=(1,4)(2,3), z_{t_{p}+3}=(1,3)(2,4) \text {. }
$$

If $y$ mates $z_{t_{p}+1}, z_{t_{p}+2}$ and $z_{t_{p}+3}$, then $y$ is a $p$-cycle. But then one of the groups $\left(z_{i}, y\right\rangle$ is soluble $\left(i \in\left\{1, \ldots, t_{p}\right\}\right)$, and so is not $A_{p} \cdot$

It is'quite possible that $A_{p}$ has spread as much as $t_{p}+2$ for good 
primes $p$, but this seems to be a very difficult result to establish.

\section{References}

[1] Г.Я. Бннбер [G.Ya. Binder], "О вдонении элементов энанопеременной группы четной степени в двухзлементный базис" [The inclusion of the elements of the alternating group of even degree in a twoelement basis], Izv. Vyšs. Učebn. Zaved. Mat. 1973, No. 8 (135), $15-18$.

[2] J.L. Brenner and James Wiegold, "Two-generator groups, I", Michigan Math. J. 22 (1975), 53-64.

[3] H.S.M. Coxeter and W.O.J. Moser, Generators and relations for discrete groups (Ergebnisse der Mathematik und ihrer Grenzgebiete, 14. Springer-Verlag, Berlin, Göttingen, Heidelberg, 1957).

[4] Ulrich Langer, "Erzeugende endlicher Iinearer Gruppen" (Dissertation, Universität Hamburg, Hamburg, 1977).

[5] Peter M. Neumann, "Transitive permutation groups of prime degree, II: a problem of Noboru Ito", Buzl. London Math. Soc. 4 (1972), $337-339$.

[6] Abraham Sinkov, "Necessary and sufficient conditions for generating certain simple groups by two operators of periods two and three", Amer. J. Math. 59 (1937), 67-76.

[7] Alan Williamson, "On primitive permutation groups containing a cycle", Math. 2. 130 (1973), 159-162.

10 Phillips Road,

Palo Alto,

Cal ifornia 94303,

USA;

Department of Mathematics,

University College,

Cardiff CFI IXL,

wales. 